\title{
EXECUTION ANALYSIS OF SPATIAL DATA STORAGE INDEXING ON CLOUD ENVIRONMENT
}

\author{
KARTHI $\mathrm{S}^{*}$ AND PRABU $\mathrm{S}^{\dagger}$
}

\begin{abstract}
Cloud computing overcome the GIS issues are huge storage, computing and reliability. Cloud computing with SpatialHadoop framework gives high performance in GIS. This paper presents spatial partition, global index and map reduce operations were studied and described in detail. Bloom filter R-tree index in the Map-reduce for providing more efficiency than the existing approaches. The BR-tree index on Map-Reduce is implemented in SpatialHadoop process that reduces intermediate data access time. Global index decreases the number of data accesses for range queries and thus improves efficiency. It is observed through experimental results that the proposed index along cloud environment performs better than existing techniques.
\end{abstract}

Key words: Spatialhadoop, BR-tree Index, Global Index, Map Reduce, Cloud Computing

AMS subject classifications. 68M14, 97R50

1. Introduction. Geographical Information System (GIS) presently we have accumulated huge geospatial data which are expanding and updating every day. GIS is utilized to catch, store, and process, analyze and display the present geospatial data. A fruitful GIS stage is not just ready to deal with vast data with complex properties, additionally gives huge data process and execution, and other computational issues. The GI [26] confront challenges in computing intensity, data intensity and concurrent access intensity. These difficulties require the preparation of a figuring framework that can better bolster revelation, give scalable and concurrent access. The cloud environment gives possible and flexible solution for the GIS issues.

A Spatial database remains as databank, and it is enhanced to keep and question information this is associated with objects in area, which includes factors, traces, polygons etc.... Though normal databases is detain to diverse numeric and personality kinds of statistics, supplementary functionality desires to be delivered for records to system latitudinal statistics types. Spatial facts stand the numerical connection between people, region, and activities. This facts can explicitly illustrate what's taking place (in which, why and how) to show the perception and effect of the beyond, the present and the (probable) destiny [1]. The proliferation of cellular programs and the huge of hardware sensing devices boom the streamed information towards the web hosting statistics-centers. This boom reasons a flooding of records. Taking blessings from these big dataset stores is a key point in growing deep insights for analysts for you to beautify gadget productiveness and to capture new commercial enterprise opportunities. Spatial analysis the crux of GIS as it includes all the alterations, manipulations, and strategies that may be carried out to geographic statistics to add fee to them, to aid decisions, and to show patterns and anomalies that aren't at once obvious. Spatial evaluation is the technique via which we flip uncooked data into beneficial information. The time period analytical cartography is now and again used to refer to methods of analysis that may be carried out to maps to make them more beneficial and informative. There are masses of approaches spatial facts can help and aide in the regular lives of all of us. The utilization of spatial information is indexed as underneath:

1. Satellite pix deliver daily weather reviews and provide farmers with facts for precision agriculture

2. Convert the integral to a linear combination of integrals of products of B-splines and provide a recurrence for integrating the product of a pair of B-splines.

3. Airborne infra-red scanners tune our bushfires

4. Ambulance message services

5. Global positioning structures divulge the region of hundreds of vans and taxis

6. Real estate income use geographic records systems

7. All styles of mapping.

The spatial information enterprise is a component of the broader facts technology sector [3] and has scientific and technical hyperlinks to all different disciplines along with environmental technology, engineering, pc technology,

* School of Computer Science and Engineering, VIT University, Vellore, TN, India

${ }^{\dagger}$ School of Computer Science and Engineering, VIT University, Vellore, TN, India 
fitness shipping, logistics, planning, useful resource control and electronics.

1.1. Data categories in spatial warehouse. The maximum recycled the classification to signify the issues of spatial enquiry consider three sorts of statistics:

Points or Events Occurrences expressed via single value identified as factors in zone, denominated theme tactics. Some samples are: sickness occurrences, crime spots, and the localization of botanical species.

Constant surfaces predicted can be beginning a hard and fast of vicinity examples which are regularly or erratically disbursed. Typically, this kind of statistics consequences from herbal sources studies, which incorporates topographical, biological, phytogeography, environmental and pedagogical charts. Zones with Calculations and Aggregation Tolls - it includes information linked to population studies, like census and fitness information [10], and which is probably initially mentioned to people located in precise elements in universe. For secrecy motives those information are aggregated in analysis gadgets, usually enclosed thru locked polygons (postal addressing zones, census tracts, municipalities). The symmetrical representations used consist of the subsequent alternatives: 2D Points: It is a well-ordered pair of $\mathrm{x}, \mathrm{y}$ values of three-dimensional directs. A factor suggests that vicinity of incidence of an occasion, like in the case of mortality via the use of outside causes. Polygons: It is a hard and fast of methodical pairs values that indicates $\mathrm{x}, \mathrm{y}$ values of three-dimensional directs, in this type of method that the ultimate opinion is same to the prime for this reason forming closed vicinity within the aircraft. In the most effective situation, every polygon delimits a person item within the most famous case; a man or woman area of interest may be delimited via numerous polygons $[11,14,15]$. Samples: encompass with ordered pairs of $\mathrm{x}, \mathrm{y}$, and $\mathrm{z}$ values in which contains the $\mathrm{x}, \mathrm{y}$ pairs mean the physical coordinates and then $\mathrm{z}$ suggests that the price of the premeditated singularity aimed at that location of zones. Typically the examples are related to area of studies, collectively with geochemical, oceanographic and then geo-physical records. The idea of a example can be widespread to the event of numerous dimensions on the identical area. Regular Grid: It is an environment in which every part is related towards a numeric rate. In this matrix, we can calculate the points which associated with a place on the ground floor. Preliminary from a initial coordinates typically noted the inferior left corner of the 10X30 matrix, and using spacing in equally to the straight and vertical directions. Image: It is in the form of matrix in which every detail is related to a numeral value (commonly from 0 to 255 variety), recycled for conception [14]. This type of condition is used to the photo presentation of a normal grid. The statistical standards in the network are ascended in shape with the production variety of the image; the superior values stand to be proven in sunnier gray shades, and then decrease in duskier gray qualities. Maximum of the geographical information systems provide the ability of providing a fixed grid in the system of a picture (popular shades or in black \& white), through a conversion that may remain computerized or measured via purchaser. Three essential shapes of spatial facts is proven in Fig. 1.1.

Database systems exercise directories to unexpectedly analyze look of values and the manner that most databases index records isn't always maximum fulfilling for latitudinal queries. Instead, three-dimensional databases practice a spatial catalog to hurry up database processes. Spatial directories are used by spatial record to enhance spatial queries [17]. Directories used by non-spatial records cannot efficaciously cope with landscapes consisting of how a protracted manner factors varies and whether or not and also points collapse inside a longitudinal vicinity of interest.

2. Related Work. Analyzed indexing and question processing in spatial statistics. Indexing strategies are used to boom the velocity of the information retrieval. In the spatio-temporal area the information continuously increases over a time and transferring item dispatched their positions. The principal drawback of spatio-temporal processing is maintaining all of the updates are not possible. The maximum of the indexing methods [1] are best supports few queries and beyond, present and destiny indexing methods shape are very complicated because it is integrated exclusive indexing methods. Interval bushes aren't in particular designed for handling unsure statistics; however one-dimensional uncertain items may be treated as durations by way of using their PDF endpoints. Both indices use a number one tree for layout and secondary structures to save the gadgets at each node, but one has a dynamic number one tree as opposed to a static one. However, the downfall of each interval indices is that if many uncertainty periods overlap with the question intervals endpoints, then few gadgets are pruned from the quest, and a number of times are wasted in calculating chances. The shape of the indexing strategies are very complex so performance is decreased and principal expectation of real time utility is concurrent updation, it isn't supported with the aid of maximum of the indexing strategies. Real time 


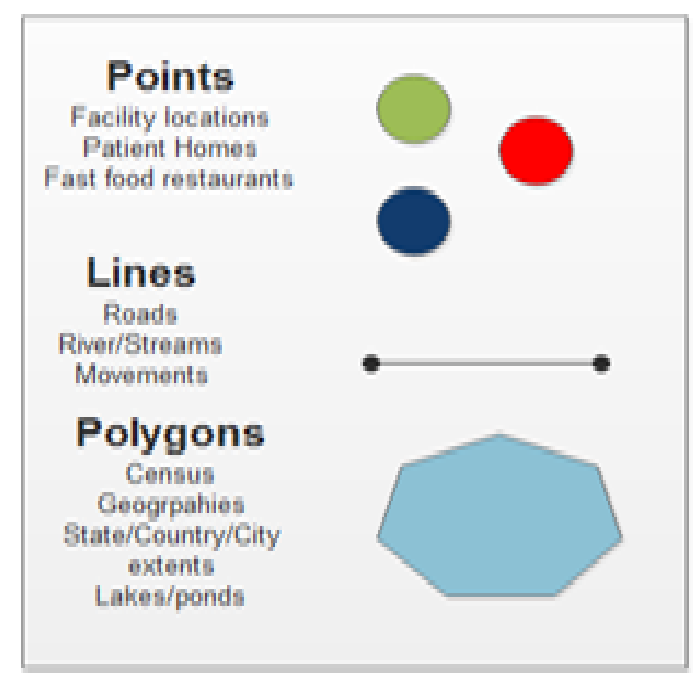

FIG. 1.1. Shapes of Spatial data

application are need comparable object locate and grouping this is also now not possible in exiting indexing techniques and eventually however now not least all of the query processing techniques aren't helps to all the kinds of indexing strategies and queries.

Analyzed spatial statistics control in cloud environments. Principal use an $\mathrm{R}+$-tree [3] to share the facts and the frames inside with go away nodes of the graphic index are preserved as active grids. First, may want to get stability among the grid scopes and then the instances of grid entrees by regulating the 2 limitations, $\mathrm{N}$ besides $\mathrm{n}$, contains of the $\mathrm{R}+$-tree. Additional, in comparison through different variations with the $\mathrm{R}$-tree, the leaf nodes ensure that which is not overlay every feature, and consequently it's distant a assistance as there is no repeated recovery of the equal statistics from special solutions and it remains simple to outline distinctive sources for every rectangle of a leaf child. Moreover, the one task is the manner to layout the vital thing terms of these networks to help effectual inquiries on BigTable manipulates schemes. We positioned the developments of CDMs equally follows: which consumes a quick (key,value) are looking for and it remains to be rapid with Image keys and it can be ordered thru a dictionary format. Based on those traits, we recommend a method to define the critical factor call of a network to help effective queries.

Presented polygon based spatial statistics assessment with map reduce. Geographic Information System which is an expedient intended to seizure, save, work, examine, manipulate, and gift completely styles of topographical facts. Spatial evaluation is a terrific function that is combining with GIS. By way of one vital process in three-dimensional evaluation, polygon overlap, that's a complex geometric set of regulations, combines the spatial and characteristic statistics of dual enter map covers [2]. In such a process, every polygon of a cover is blended with some other layer in couple to reap the cease end result. Classically, particular thematic covers of the identical location are occupied and covered any on pinnacle with opportunity to yield an outcome layer. Increasingly, the dimensions range, and replace charge of a few longitudinal datasets surpasses the ability of three-dimensional computing generation. In a diffusion of times, overlap assessment will become a inefficient venture as commerce with big dimensions of three-dimensional statistics is needed. The computer GIS software normally takes times to perform overlap of this large spatial informations. Such consumption on time is undesirable for lots packages, specifically for actual time coverage choices which encompass predicting which homes would be broken through manner of a transcontinental storm.

Analyzed spatial facts processing using Map-Reduce which is a programming version and computing platform well ideal for parallel computation. In Map Reduce, a application includes a map feature and a reduce function that are consumer-described. The input facts layout is software particular, and is designated by way of the consumer [5]. At first, spatial splitting is adopted to distribute information to all nodes as even as possible, 
and then strip-based two path sweeping set of rules can accelerate the computation instead of spatial index in conventional spatial packages. Finally, pending documents and redundant statistics are used to deal with the relationship between the spatial items. And adopted Map Reduce to process megastar catalog cross certification in astronomical discipline. In order to take full use of the Map Reduce platform, it's far better to make complete issues of parallel algorithm plant. In this section, Map Reduce is carried out to pass-certification, and then in comparison with conventional PostgreSQL DBMS. As a simple and quintessential step, the astronomical cross certification is facing a data avalanche. With the of entirety of latest sky survey tasks and effective telescopes, present day go-certification methods cannot be done on call for large scale astronomical information units. In this paper, Map Reduce framework is delivered to clear up this trouble. The mapping of move-certification algorithm on Map and Reduce phases is carefully taken into consideration. Performance assessment has shown that the Map Reduce-based totally move-certification can outperform the conventional one on PostgreSQL. As our expertise, it is the first attempt to undertake Map Reduce for astronomical go-certification problem.

Implemented partition techniques in spatial hadoop. SpatialHadoop [4] offers a prevalent indexing set of regulations which grow to be recycled to implement network, R-tree, and R+-tree primarily founded for partitioning. This paper spreads preceding observe by way of manner of introducing 4 new partitioning strategies, Hilbert curve, Z-curve, K-d tree and Quad tree and have a look at entirely the seven strategies. The partitioning section of the indexing procedure turns in three steps, in which the initial step is regular and then closing dual steps are custom designed for every partitioning methods. The initial step calculates amount of favorite dividers $\mathrm{n}$ based totally mostly on record length and the HDFS chunk functionality which may be each fixed for complete partitioning techniques. The distribution technique allocates an item to precisely one overlying cell and then the cellular desires to stand increased to surround all controlled statistics. The repetition method eludes growing cells with the useful resource of replicating every data to all overlying cells and query processor consumes to lease a reproduction evading approach to explanation for simulated facts. While range query executed similarly on all of them, we showed that they will be tuned with device parameters which encompass block period in line with the question paintings load. We additionally confirmed the overall performance of spatial be a part of is strongly correlated with the fee of Q1 (average region of partitions) and placed that Quad tree outperformed unique techniques being experimented.

3. Existing Methodologies. The amount of data in spatial databases is developing as greater records are made to be had. Spatial databases in particular store distinct varieties of facts: raster records (satellite/aerial virtual pictures), and vector information (factors, polygons, lines). The complexity and environment of threedimensional databases types them first-class for smearing similar processing [20]. The cutting-edge methods are managing Map-Reduce framework in indexing systems like as $\mathrm{R}$ tree. There are different variation in $\mathrm{R}$-tree that is used of static databases and another one for dynamic databases. The overall performance of R-wood relies upon at the exceptional of the set of rules that groups the data frames on a node.

3.1. Constructing R-Tree with Map Reduce. Let D be a spatial data set composed of devices. Each item i has attributes $j$ i.Id,i.P $\dot{i}$, in which i.Id is referred as the items particular identifier and i.P is also known as objects region in particular three-dimensional domain; different features are possible, but we can supply interest to those quality for R-Tree production cause. R-Tree is consist of minimum bounding rectangles (MBRs) that are created primarily founded on the devices spatial characteristic of i.P. i.Id is used as references to items saved within the RTree in form of leaves. In a main, the spatial objects are divided into nodes. Then, each leaf is processed to generate a minor R-Tree. Finally, the minor R-Trees are combined into the last R-Tree. The principal degrees are carried out in Map Reduce, at the equal time because the remaining segment does not involve large number of computational steps, therefore it is far implemented consecutively out of entries in the cluster [17]. The levels in $\mathrm{R}$ tree index with Map-Reduce are shown in Fig. 3.1. The Major disadvantage with $\mathrm{R}$ tree with Map-reduce is aid only variety queries for future data retrieval in spatial records processing.

3.2. Hillbert R-Tree with Map Reduce. The basic presentation of R-tree relies upon at the great set of rules that groups the frames on a node, at the identical as Hilbert curve [23] which contains the quality of spatial cluster belongings. Hilbert R-tree is extension of Hilbert space-filling-curves approach and in particular the Hilbert value to execute a linear ordering on the information rectangles; then, it negotiates the sorted listing, assigning each set of values at the equal node [20] in the linear ordering, and maximum likely in the 
Phase 1 : Partitioning Function Computation

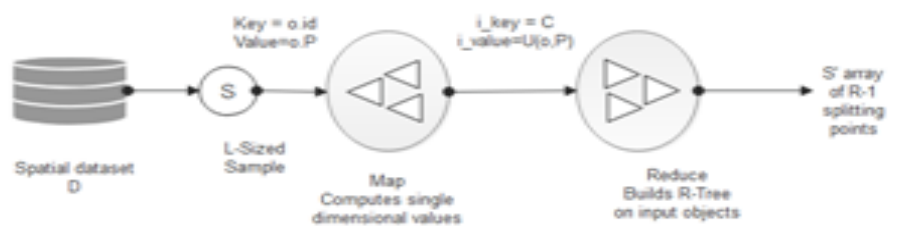

Phase 2: R - Tree construction

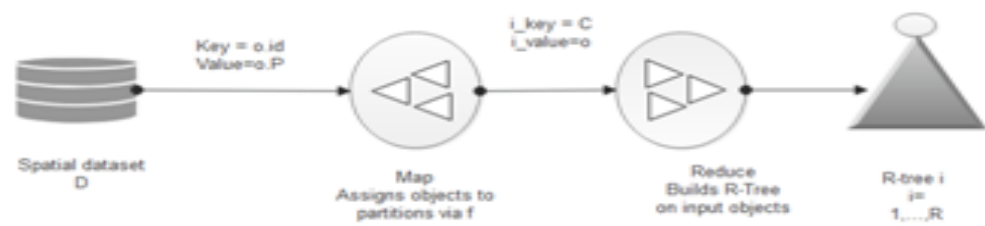

Phase 3 : R-Tree consolidation

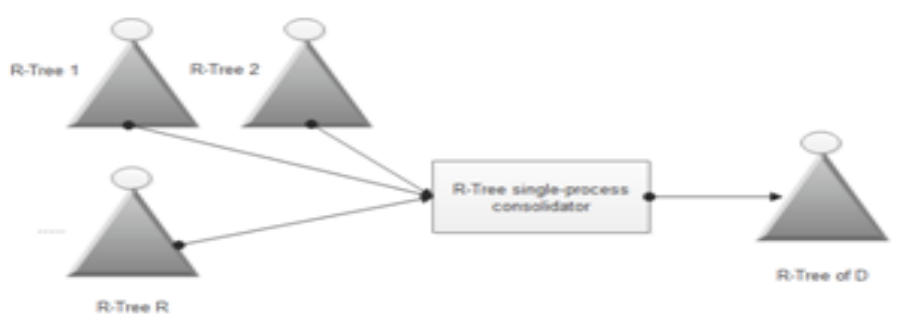

FIG. 3.1. Phases in R-tree indexing in Map-Reduce framework

neighborhood area; therefore, the following R-tree nodes could have the slighter regions. The Hilbert R-tree consists of following structure.

The algorithm of build Hilbert R-tree is given as follows:

- Step 1 Compute the Hilbert cost for each nodes in database.

- Step 2 Categorize statistics data and align as ascending Hilbert values.

- Step 3 Build the R-tree from bottom to up recursively in step with the directive of Hilbert values

The assumption of the algorithm is that the data are static or the frequency of amendment is short. The approach is an easy heuristic for building an R-tree with 100 place of utilization, which at the equal time could have as proper reaction time as viable [18]. In precise, the set of guidelines might be very suitable for parallel bulk-loading processing.

Preliminaries of Hilbert Cost: The Hilbert cost of a node is described due to the fact the Hilbert fee of its center. The manner of calculating the Hilbert value for a data rectangle is split into two steps:

- Step 1 Compute the grid cell that the intermediate of a data are plotted in area

- Step 2 Compute the Hilbert value of the every grid area

Figure 3.2 demonstrates some nodes ordered in a Hilbert R-tree which has the following structure. The Hilbert values of the facilities are the records near the $\mathrm{x}$ symbols (proven simplest for the determine node II). The LHVs are in [brackets].A leaf node contains a most $\mathrm{Cl}$ entries each of the shape ( $\mathrm{R}$, obj id) in which $\mathrm{Cl}$ is the potential of the leaf, $\mathrm{R}$ is the MBR of the real object (xlow, xhigh, ylow, yhigh) and obj-identity is a pointer to the item description document. The vital distinction between the Hilbert R-tree and the $\mathrm{R}^{*}$-tree is that non-leaf nodes also include facts about the LHVs (Largest Hilbert Value). Thus, a non-leaf node in the Hilbert R-tree contains a most Cn entries of the form (R, ptr, LHV) in which Cn is the capacity of a non-leaf node, $\mathrm{R}$ is the MBR that encloses all of the kids of that node, ptr is a pointer to the kid node, and LHV is the 


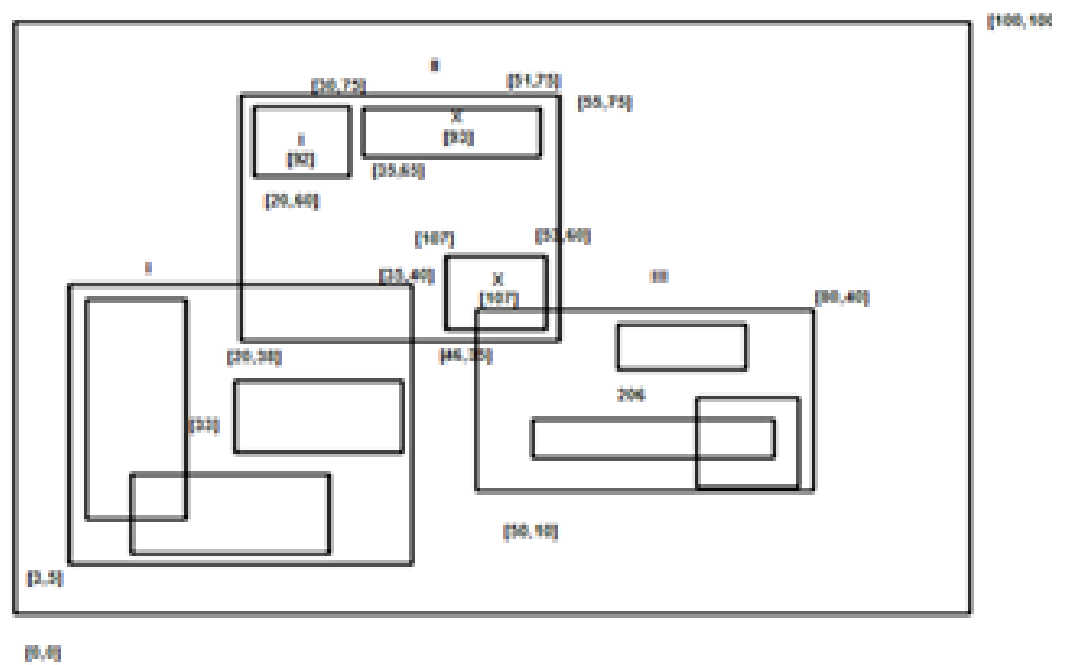

FIG. 3.2. Hilbert $R$ tree structure

largest Hilbert price the various facts rectangles enclosed by means of using R. Notice that because the non-leaf node alternatives one of the Hilbert values of the children to be the price of its non-public LHV, there isn't extra rate for calculating the Hilbert values of the MBR of non-leaf nodes.

4. Rapid Indexing Scheme for Spatial Data Processing in Cloud Enviroment. This work integrates a BR-tree index in MapReduce that results in a parallel B-Tree index. The significance of MapReduceHadoop and indexing in the Hadoop is described below.

4.1. Map Reduce. Large extent of facts has led to adoption of parallel processing. It provides a green processing over a fixed of participating pc machines. It changed into expected that parallel processing might offer new ways of thinking about the prevailing concept of programming language, working machine and storage system for massive dispensed systems. Parallel processing is complex, however many frameworks have evolved that offer parallel processing the use of abstraction to simplify matters. Hadoop, a Java implementation of Map Reduce, has emerged as one such framework. It works on key-price storage concept and has specifically two components, Map Reduce and HDFS. Map Reduce part of the Hadoop encapsulates all info of parallel processing from customers and they get a totally simplified framework for programming. Map Reduce has come to be very famous for parallel dispensation of subjective information. It is mechanism and consists of divide and-conquers technique and pauses a calculation into sub-calculations over established of computer systems in a group that featured as equivalent. Every smaller calculation is dealt with independently and the cease result of the calculation is lower back lower again at a vital issue. A Map Reduce application takes information in the key-cost shape from HDFS and techniques it. It works through well-known features: map and decrease. The map feature accepts input statistics inside the key-price shape and produces a few intermediate facts. Once the map characteristic is completed, reduce feature starts off evolved. The reduce characteristic takes as input the intermediate facts having identical key and produces output facts that is written returned to HDFS. Many map and reduce functions paintings concurrently on one of a kind splits of the enter dataset in HDFS. A huge amount of records switch takes place among the map and decrease features whilst intermediate records are produced. A combiner function can be used to lessen intermediate facts and statistics transfers with the aid of aggregating information on the premise of intermediate key.

4.2. Indexing Scheme With Mpa-Reduce. Initially, MapReduce has been used for large scale records in depth packages for records retrieval from semistructured and unstructured facts. The MR-LSI algorithm retrieves scalable statistics from the unstructured files quite effectively. It has been used correctly in the area of based statistics for expressing queries. Hadoop by default shops facts in key-price form and distribution of 


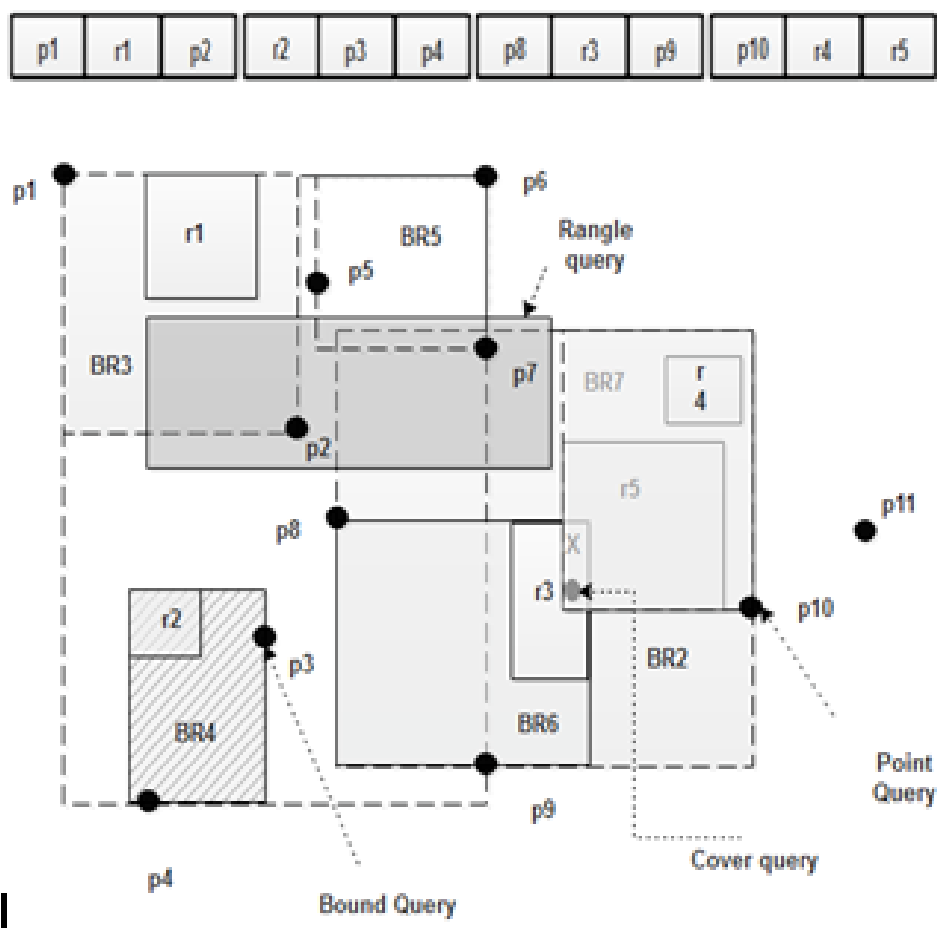

FIG. 4.1. Bloom filter Framework

data over computing nodes takes region via a hash function implemented on keys. The hash feature acts as the primary index for speedy accessing records. However, search over non-key records calls for a secondary index for immediate gaining access to. In the absence of a right secondary index, map tasks are wasted on the undesired dataset. A proper indexing over the input dataset higher organizes the dataset, and minimizes computation. It utilizes map duties for only the desired and filtered dataset, and therefore, improves query resource time and saves machine assets.

4.3. Constructing BR-Tree Indexing in Data Node. Bloom filters base R-tree (BR-tree) in which bloom filter out is integrated to R-tree node. BR-tree is basically R-tree shape for assisting dynamic indexing. In it each node maintains range index to indicate characteristic of gift object. Range query and cowl question supported as it shop object and form of it together. A Bloom clean out is a area-green statistics shape to shop an index of an object and may represent a hard and fast of items as a piece array the usage of numerous impartial hash abilities. BR-tree node is combination of R-tree node and Bloom clean out. BR-tree is likewise loading balanced tree. Overloaded bloom clears out produce excessive duplicate effective probabilities. It reconfigures the multidimensional variety using bounding boxes to cowl item. BR-tree help bound question the first index structure to speak about the certain question. Bound query result into range information of multidimensional function of a queried object. It is not trivial due to the fact BR-tree maintains benefit of Bloom clean out and R-tree each. It mixes the queries like bound question and range question after thing question end result is incredible. BR-tree continues consistency between queried records and the characteristic sure in an integrated shape so that fast point question and accurate sure question viable. The number one format of Bloom clear out is tested in Fig 4.1.

Bloom filters are area-green probabilistic data systems to begin with idea to test whether or not or now not an detail is member of a set or not. Bloom filters have a one hundred keep in mind charge, because faux remarkable suits are viable, whereas fake horrible fits are not possible. It has been validated that Bloom filters are very useful gear within Map Reduce obligations, thinking about that, given the truth that they keep away 
from fake negatives, they allow for doing away with beside the point facts at some point of map levels of Map Reduce duties, as a result configuring themselves as a very dependable area-green answer for Map Reduce. The gain of Bloom clean out is space overall performance. The length of the Bloom clear out is constant nevertheless of the variety of the features $\mathrm{n}$, however there may be a tradeoff among $\mathrm{m}$ and the false high pleasant opportunity p. The opportunity of a fake high great after placing $n$ factors can be intended as follows:

$$
p=\left(1-(1-1 / n)^{k m}\right)^{k} \approx\left(1-e^{(k m / n)}\right)^{k}-\operatorname{Eqn}(1)
$$

\subsection{Execution of proposed work.}

1. Job suggestion. If an activity is acquiesced, $\mathrm{m} 1$ is the map task for $\mathrm{R}, \mathrm{m} 2$ is map task for $\mathrm{S}$, and $\mathrm{r}$ reduce duties are created. A task contains all crucial data to be track on a task tracker which incorporates the attention formation and the area of the matching input/output records.

2. First map phase. Activity tracker allocates the m1 map obligations or the reduce duties to lazy assignment trackers. The map task tracker reads the entire data and split it for the assignment, converts it to key or value pairs, and then performs the map feature for the every input dataset.

3. Local filter construction. The in-between pairs comprised of the map function are separated into $r$ spilts, which may be dispatched to $\mathrm{r}$ project trackers respectively. Moreover, Bloom filters are built at the bases in every partition and referred as filters in the name of community filters due to the fact they may be constructed for simplest the in-between effects in a sole undertaking tracker. If a venture tracker runs more than one map duties, it merges the close by filters of every assignment and then continues merely $\mathrm{r}$ filters.

4. Global filter merging. After all m1 map obligations are whole, the process tracker indicators all challenge trackers to ship the nearby filters through heartbeat messages. Then, all assignment trackers send their community filters to the process tracker, and then process tracker concepts the overall filters to the dataset R. Next, the process tracker sends the overall filters to all project trackers. Until constructing and transmitting the global filters that are whole, the map tasks to the dataset $\mathrm{S}$ aren't allocated.

5. Additional map segment. Then job tracker allocates the $\mathrm{m} 2$ map duties or the enduring lessens duties with task trackers. Task trackers track the allotted project with the established international filters. The enter key or cost pairs cant be set inside the global filters for filtered out.

6. Reduce segment. This step is the same as the lessen section in Hadoop. A reduce task tracker reads the corresponding intermediate pairs from all map task trackers the usage of far flung system calls. It kinds the all intermediary pairs and runs the lessen characteristic. Final output outcomes are written inside the given output course. We have made modifications to the layout of Hadoop. First, we includes map task inside the instruction of the information. Second, include assemble Bloom filters at the build enter in allotted fashion to clear out the probe enter. The proposed format is shown in Fig.4.2.

The procedure of MR-BR-Tree takes inputs as various data types and produce output as spatial index. The pseudo code of procedure is described as follows:

\section{Algorithm 1: Rapid Indexing for Spatial Data (RISD)}

Input: Spatial Data points or Lines or Polygons $\mathrm{D}=\left\{d_{1}, d_{2}, \ldots, d_{n}\right\}$

Output: Index tree $I_{E}$

Step 1: for all data types (d) in D do

Step 2: spilt data into partitions $P_{i}$

Step 3: Normalize $d_{i}$

Step 4: Compute (key,value) c of $P_{i}$ using BR tree and add C

Step 5: end for

Step 6: I=BuildBRtree() as $\operatorname{Eqn}(2)$

Step 7: Send Index $I_{E}$ to admin

In this partition algorithm, we can read the spatial datasets as input they can be as many types. 


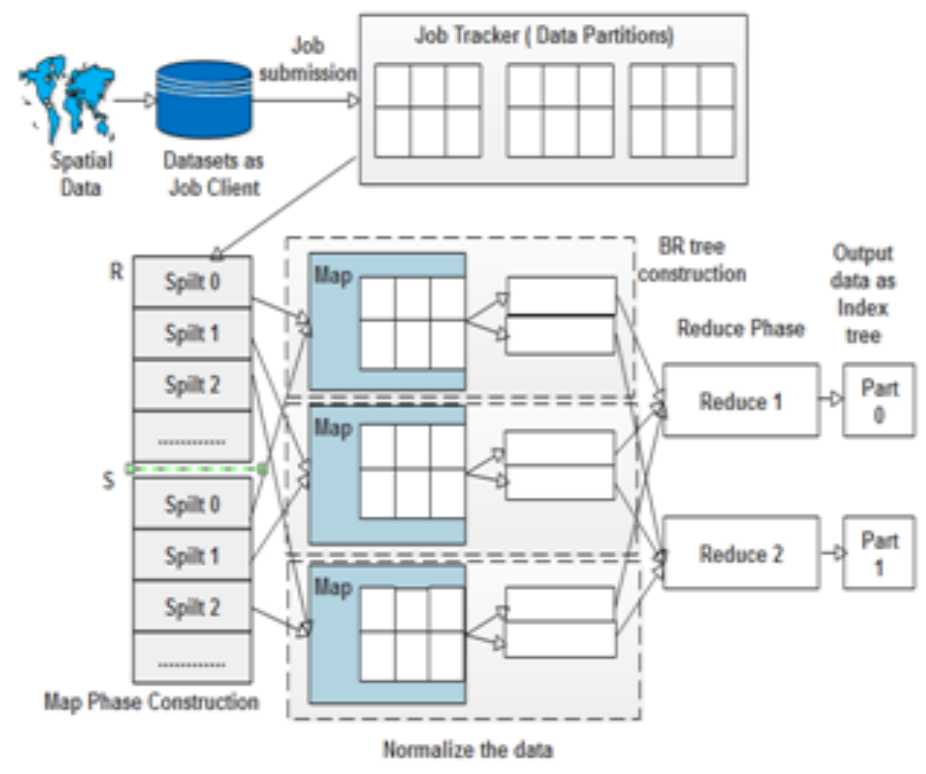

Fig. 4.2. Proposed Framework

Input: Spatial points D

\section{Algorithm 2: Partitioning algorithm}

for all object Obj in mapper phase do

if $(D[i-1]<O b j$. Value $\leq D[i])$

Send Obj to reducer $\mathrm{i}$

end for

This guarantees that the result of reducer $i$ is less than the result of reducer $i+1$. Formerly, every reducer categories each map records directly and writes them into single partition task, the ones looked after partition documents shape a globally taken care of document. Each spatial statistics factor di in D is normalized to [0, 1$]$.

4.5. Build Bloom filter R Tree:. While a task tracker runs a map undertaking of the build the data and send this data as input, and it makes the Bloom filters as intermediate records made out of the challenge. A Bloom filter is shaped for every map output partition allocated for each reduce assignment, and therefore the general wide variety of the Bloom filters is the amount of lessen obligations. When more than one map duties are run on a undertaking tracker, the challenge tracker merges each Bloom filters from the responsibilities to keeps simplest unique kind of Bloom filters. And call this traditional of Bloom filters as close by filters. When complete map tasks aimed at the construct input are comprehensive, the pastime tracker has to fold all neighborhood filters to collect the global filters. And upload Task Tracker Action lessons, known as Local Filter approach and Receive Global Filter Action, for the Global filter approach.

The task tracker shows the Local filter action is in the form of heartbeat reaction to entire challenge trackers, and that they direct the task tracker their native filters. The activity tracker joins all of the nearby strainers to construct the worldwide sifters the usage of bitwise OR processes, and mentions the action as Receive Global Filter Action by means of the global filters in the heartbeat message towards total task trackers. The conversation price aimed at the filter for analyzed Cf is

$$
C f=2 c t \cdot n \cdot r e \cdot t k-\operatorname{Eqn}(2)
$$

wherein ct is the amount to allocation data from single node to another, $\mathrm{n}$ remains the dimensions of Bloom filter, re is the wide variety of lessen tasks, and tk is the amount of challenge trackers. The coefficient 2 is accelerated for the reason that neighborhood filters and with the global filters are transmitted among the task tracker and the project trackers. Our proposed structure significantly improves the execution time of queries. 


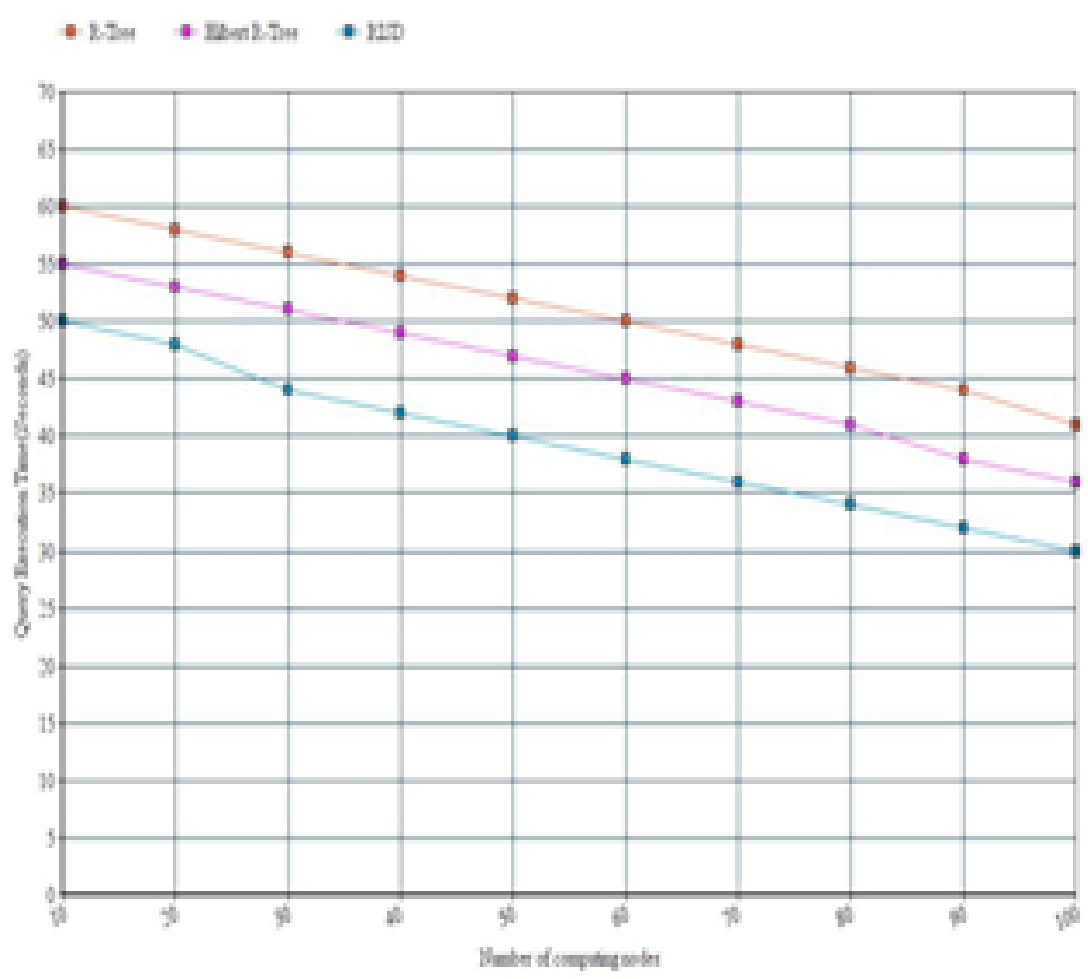

5. Experimental Results. Experiments are carried out for performance evaluation of search query on MR-BR-Tree indexed MapReduce The search query execution time of the R, Hilbert R and RISD Index on spatial data. The time taken to distribute the partitioned data onto cluster nodes and managing a global index of the master node is almost similar in all the three approaches. The query execution time shrinkages as the size of cluster upsurges. It remains due to parallelization of query. The query completing time is composed of the amount of time elapsed in map and reduce phases. The search query is decomposed into sub-queries that run in parallel, and consequently, execution time decreases. Initially, there is a small rise in execution time when second and third node is added to the cluster. It is due to increased sorting and shuffling in intermediate stages that overcomes the gain in performance due to fast computation achieved with parallelization. But later on, the performance gain achieved with parallel computation exceeds the burden of sorting and shuffling. Consequently, execution time decreases gradually with the addition of nodes in the cluster. In our proposed RISD framework, the index is built only one time on the complete dataset and only range search queries execute on the index. The indexing of the data reduces query execution time. The Performance chart is shown in Fig 5.1. The RISD performs almost better than the B-Tree and Hilbert $\mathrm{R}$ tree in spatial datasets.

6. Conclusion. In this work we studied and implemented the performance of bloom filter R-Tree in cloud platform. The results show that when using an instance with greater resources a better performance will be gained. The Map-Reduce technology has proved very effective for large scale structured, semi-structured and unstructured data, for information processing and retrieval. In this connection, a B-Tree index, in a chainedMapReduce process, is designed and implemented. The proposed work compares various indexed data structures that include RISD, R, Hibert R of Hadoop, for range search queries. It is observed that a significant amount of time is less to build indexes in proposed system than the existing system. 


\section{REFERENCES}

[1] John, A., M, Sugumaran and R. S. Rajesh, Indexing and Query Pprocessing Techniques in Spatio-Temporal Data, ICTACT Journal on Soft Computing 6.3, 2016.

[2] Wang, Kai, Jizhong Han, Bibo Tu, Jiao Dai, Wei Zhou, and Xuan Song, Accelerating spatial data processing with mapreduce, Parallel and Distributed Systems (ICPADS), 2010 IEEE 16th International Conference on. IEEE, 2010.

[3] Eldawy, Ahmed, Louai Alarabi, and Mohamed F. Mokbel, Spatial partitioning techniques in SpatialHadoop, Proceedings of the VLDB Endowment $8.12,1602-1605,2015$.

[4] Wang, Yong, Zhenling Liu, Hongyan LiaO, And Chenguun Li, Improving the performance of GIS polygon overlay computation with MapReduce for spatial big data processing., Cluster Computing 18.2, 507-516, 2015.

[5] Wei, Ling-Yin, Ya-Ting Hsu, Wen-Chin Peng, and Wang-Chien Lee, LU-Indexing spatial data in cloud data managements, Pervasive and Mobile Computing 15 (2014): 48-616.

[6] Rajashekhar M. Arasanal and Dannish U. Rumani, Improving MapReduce Performance through Complexity and Performance Based Data Placement in Heterogeneous Hadoop Clusters, Distributed Computing and Internet Technology, Lecture Notes in Computer Science Volume 7753, 2013, pp. 115-125, 2013.

[7] Performance Measurement of a Hadoop Cluster, , http://www.acma.com/acma/pdfs/AMAX Emulex Hadoop Whitepaper.pdf (Accessed on December 20, 2015

[8] Jeffrey Dean and Sanjay Ghemanat, MapReduce: SimplifiedDataProcessing on Large Clusters, Magazine Communications of the ACM - 50th anniversary issue: 1958 2008, Vol. 51 Iss. 1, pp. 107-113, 2008.

[9] http://sortbenchmark.org/Yahoo2009.pdf (April 2009) (Accessed on February 22, 2016).

[10] M.K. Aguilera, W. Golab, and M.A. Shah, A practical scalable distributed b-tree, PVLDB, Vol. 1, Iss. 1, pp. 598-609, 2008.

[11] Burhan Ui. Islam Khan, Rashidah F. Olanrewaju, Hunain Altaf and Asadullah Shah, Critical insight for MapReduce optimization in Hadoop, International Journal of Computer Science and Control Engineering, Vol. 2, Iss. 1, pp. 1-7, 2014.

[12] Matei Zaharia, Andy Konwinski, Anthony D. Joseph Randy Katz and Ion Stoica, Improving MapReduce Performance in Heterogeneous Environments, In Proceedings of the 8th USENIX conference on Operating systems design and implementation, pp. 29-42, USENIX Association Berkeley, CA, USA 2008.

[13] Jimmy Lin And AleK Kolcz, Large-scale machine learning at Twitter, Proceedings of the 2012 ACM SIGMOD International Conference on Management of Data, Scottsdale, AZ, USA, pp. 793 804, 2012

[14] Fan Zhang, Junwei Cao, Samee U. Khan, Keqin. Li and Kai Hwang, A task-level adaptive MapReduce framework for real-time streaming data in healthcare applications, Future Generation Computer System, Vol. 43 44, pp. 149 160, 2015.

[15] David A. Patterson, Technical perspective: the data center is the computer, Communications of the ACM, Vol. 51, Iss.1, pp. 105-105, 2008.

[16] Eric Anderson and Joseph Tucek, Efficiency matters!., ACM SIGPOS Operating Systems Review, Vol. 44, Iss. 1, pp. 40-45, 2010.

[17] Feng Li, Beng Chin Ooi, M. Tamer Ozsu and Sai Wu, Distributed Data Management Using MapReduce, ACM Computing Surveys, Vol. 46, Iss. 3, Article No. 31, 2014.

[18] H.V. Jagdish, B.C. OoI, AND Q.H. Vu. BATON, A balanced tree structure for peer-to-peer networks, In Proceedings of the 31st International Conference on Very large data bases, VLDB Endowment, pp. 661-672, 2005.

[19] F.N. Aftari and J.D Ullman, Optimizing joins in a MapReduce environment, In Proceedings of the 13th International Conference on Extending Database Technology, EDBT, pp. 99-110, 2010.

[20] Sai Wu, Dawei Jiang, Beng Chin Ooi, and Kun-Lung Wu, Efficient b-tree based indexing for cloud data processing, Proceedings of VLDB Endowment,Vol. 3, Iss. 1, pp. 1207-1218, 2010.

[21] Ian H. Witten, Alistair Moffat, and Timothy C, Bell. Managing Gigabytes: Compressing and Indexing Documents and Images, Morgan Kaufmann, ISBN 1558605703, 1999.

[22] Steffen Heinz and Justin Zobel, Efficient single-pass index construction for text databases, JASIST, Vol. 54, Iss. 8, pp. 713-729, 2003.

[23] Anthony Tomasic and Hector Garcia-Molina, Performance of inverted indices in shared-nothing distributed text document information retrieval systems, Proceedings of PDIS, pp. 8-17, 1993.

[24] Mike Cafarella and Doug Cutting, Building Nutch: Open source search, ACM Queue, Vol. 2, Iss. 2, pp. 54-61, 2004.

[25] Mark Lillibridge, Kave Eshghi, Deepavali Bhagwat, Vinay Deolalikar, Greg Trezise and Peter Camble, Sparse indexing: large scale, inline deduplication using sampling and locality, In proceedings of USENIX Conference - File and Storage Technologies (FAST), pp. 111-123, February 2009.

[26] Yang, Chaowei, Michael Goodchild, Qunying Huang, Doug Nebert, Robert Raskin, Yan Xu, Myra Bambacus, and DANIEL FAY, Spatial cloud computing: how can the geospatial sciences use and help shape cloud computing?, International Journal of Digital Earth. 4(4) pp. 305-329, 2011.

Edited by: Rajkumar Rajasekaran

Received: Jul 24, 2018

Accepted: Dec 14, 2018 